\title{
Processing used nuclear fuel with nanoscale control of uranium and ultrafiltration
}

\author{
Ernest M. Wylie $^{\mathrm{a}}$, Kathryn M. Peruski ${ }^{\mathrm{a}}$, Sarah. E. Prizio ${ }^{\mathrm{a}}$, Andrea N.A. Bridges ${ }^{\mathrm{d}}$, Tracy S. \\ Rudisill $^{\mathrm{d}}$, David T. Hobbs ${ }^{\mathrm{d}}$, William A. Phillip ${ }^{\mathrm{b}}$, Peter C. Burns ${ }^{\mathrm{a}, \mathrm{c}}{ }^{*}$ \\ ${ }^{a}$ Department of Civil and Environmental Engineering and Earth Sciences, University of Notre \\ Dame, Notre Dame, IN 46556, USA
}

${ }^{\mathrm{b}}$ Department of Chemical and Biomolecular Engineering, University of Notre Dame, Notre Dame, IN 46556, USA

${ }^{\mathrm{c}}$ Department of Chemistry and Biochemistry, University of Notre Dame, Notre Dame, IN 46556, USA

d Savannah River National Laboratory, Aiken, SC 29808, USA

*Corresponding Author: pburns@nd.edu

\section{Present Addresses}

†Department of Environmental Engineering and Earth Sciences, Clemson University, Clemson, SC 29634, USA 


\section{ABSTRACT}

Current separation and purification technologies utilized in the nuclear fuel cycle rely primarily on liquid-liquid extraction and ion-exchange processes. Here, we report a laboratory-scale aqueous process that demonstrates nanoscale control for the recovery of uranium from simulated used nuclear fuel (SIMFUEL). The selective, hydrogen peroxide induced oxidative dissolution of SIMFUEL material results in the rapid assembly of persistent uranyl peroxide nanocluster species that can be separated and recovered at moderate to high yield from other process-soluble constituents using sequestration-assisted ultrafiltration. Implementation of size-selective physical processes like filtration could results in an overall simplification of nuclear fuel cycle technology, improving the environmental consequences of nuclear energy and reducing costs of processing.

\section{Abstract Graphic}

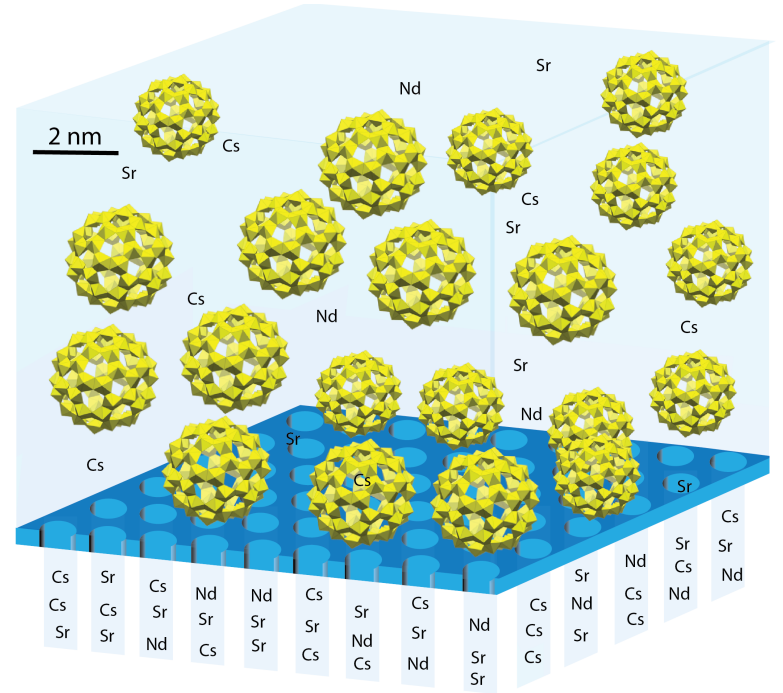




\section{Introduction}

Recovery of actinides from complex material began during the Manhattan Project for the production of nuclear weapons $[1,2]$. Since its inception, recovery technology has evolved into many industrial-scale processes used for the extraction, separation, and remediation of useful material from all phases of the nuclear fuel cycle. Solvent extraction is the most widely utilized reprocessing technology and has been industrially refined over decades, resulting in high separation efficiency [3]. It is based on the selectivity of specific ligands in an organic phase for target species in a separate immiscible aqueous phase [4-6]. This results in a large volume of corrosive, toxic, radioactive, liquid waste that presents problems regarding material storage, transport, and remediation [7-9]. Current commercial reprocessing efforts in the UK and France vitrify waste shortly after separation from the fuel and produce less liquid waste than earlier efforts, but more efficient processes would still lead to significant improvements relative to cost and environmental impact. One largely unexplored approach is the application of actinide nanomaterials in recovery processes.

New processes are being developed in an attempt to address the issues associated with liquidliquid extraction in nuclear waste processing. Ongoing research in materials science seeks to use recent advances in the growing field of nanotechnology to improve process design. Perhaps owing to the challenges of nuclear waste, including the complex chemistry of the actinides and the extreme radioactivity of the systems, emphasis remains on liquid-liquid extraction, rather than developing "blue sky” approaches that incorporate nanomaterials. As commercial fuel is composed primarily of uranium, processes tailored to manipulate uranium speciation are a clear target for these new designs [10]. A growing and diverse family of uranyl peroxide nanoclusters offers promise for the nanoscale control of uranium through various phases of the nuclear fuel 
cycle [11-13]. Manipulation of these materials on the nanoscale may allow for the replacement of complex chemical extraction with a simple, physical size-selective filtration method, resulting in the alleviation of many of the problems associated with currently utilized liquid-liquid extraction techniques. Our approach utilizes alkaline aqueous solutions with added peroxide, and builds upon earlier studies of broadly similar systems [14-16].

Here we demonstrate that uranyl peroxide clusters can be separated from chemically complex solutions that are comparable to those processed in spent fuel recycling. We report the recovery of purified uranium at moderate to high yield from the hydrogen peroxide induced oxidative dissolution of simulated used nuclear fuel (SIMFUEL) and subsequent sequestration-assisted ultrafiltration of the resulting uranyl peroxide nanocluster species. Our initial discovery of the conditions under which uranyl peroxide polyhedra spontaneously self-assemble into nanoscale cage clusters led to an extensive synthetic effort that has resulted in more than 50 distinct uranium-based nanomaterials with well defined structures [11-13]. These clusters, containing as many as 124 uranyl polyhedra and with diameters in the range of 1.5 to $4.0 \mathrm{~nm}$, form rapidly in aqueous solution, promote the dissolution of uranium-based solids, persist for many months, and are highly soluble [17-19]. The cages of these clusters are defined by uranyl ions that are bridged by peroxide groups, and in some cases by various other ligands including hydroxyl, phosphate, pyrophosphate, oxalate, nitrate, and phosphite. Many adopt fullerene topologies, including U60, which is topologically identical to C60 [20]. Our objective here is to demonstrate dissolution of SIMFUEL under ambient aqueous conditions in water with added peroxide, the self-assembly of uranyl peroxide cage clusters in the resulting solution, and the separation of these cage clusters from other constituents in solution by ultrafiltration. This process is shown schematically in Figure 1, which illustrates U60 rejection by a porous membrane. 


\section{Experimental}

\subsection{SIMFUEL preparation}

Simulated used nuclear fuel was prepared at Savannah River National Laboratory. The specific composition of the material was calculated using the ORIGEN2 code developed at Oak Ridge National Laboratory [21]. Parameters for the calculation were a burn-up of 60 GWd/MTU followed by 5 years of cooling. The total mass of the material was $53.6559 \mathrm{~g}$ mixed using the individual measured masses listed in the Supporting Information. Non-radioactive isotopes of the selected fission products were used, to facilitate handling of the material. There is no nonradioactive isotope of technetium, and neither it nor the trans-uranium isotopes were included in this study. Subsequent to mixing, the material was mechanically pressed at Savannah River National Laboratory into 10 individual $\sim 5$ g pellets and shipped to the University of Notre Dame. Upon arrival, the pellets were placed into an alumina combustion boat and sintered at $1650{ }^{\circ} \mathrm{C}$ for $6 \mathrm{hr}$ using a $48 \mathrm{hr}$ total ramp in an atmosphere consisting of $4 \% \mathrm{H}$ in Ar. After the pellets were cooled to room temperature, they were separated into individual sealed polypropylene tubes.

\subsection{Oxidative dissolution and solution preparation of SIMFUEL material}

Prior to dissolution, the pressed and sintered pellets were crushed into fine powders using a mortar and pestle. Then, $\sim 1 \mathrm{~g}$ of powdered material was treated with $2 \mathrm{~mL}$ of $2.4 \mathrm{M} \mathrm{LiOH}$ followed by $6 \mathrm{~mL}$ of $30 \% \mathrm{H}_{2} \mathrm{O}_{2}$ resulting in aqueous uranyl from:

$\mathrm{UO}_{2+\mathrm{x}(\mathrm{s})}+3 \mathrm{H}_{2} \mathrm{O}_{2(\mathrm{l})} \rightarrow \mathrm{UO}_{2}{ }^{2+}{ }_{(\mathrm{aq})}+2 \mathrm{H}_{2} \mathrm{O}_{(\mathrm{l})}+\mathrm{O}_{2(\mathrm{~g})}+2 \mathrm{OH}_{(\mathrm{l})}^{-}$ 
When aqueous uranyl reaches an appropriate concentration, the following generalized reaction occurs:

$\mathrm{UO}_{2}{ }^{2+}{ }_{(\mathrm{aq})}+(6-2 \mathrm{n}) \mathrm{OH}^{-}+\mathrm{nH}_{2} \mathrm{O}_{2(\mathrm{l})} \rightarrow \mathrm{UO}_{2}\left(\mathrm{O}_{2}\right)_{\mathrm{n}}(\mathrm{OH})_{(6-2 \mathrm{n})}{ }^{4-}{ }_{\text {(aq })}$

In the presence of excess hydroxide and peroxide, cluster fragments and ultimately fully formed nanoscale clusters will assemble in solution, with this process occurring within an hour $[18,19]$. Note that a small quantity of solid remained after dissolution, as not all of the fission products are soluble under these conditions.

After visible offgassing became minimal, the solution was removed from the reaction vessel and passed through a $2 \mu \mathrm{m}$ coarse membrane to separate undissolved solids. This solution was allowed to equilibrate for $12-18 \mathrm{hrs}$. After this period, $1 \mathrm{~mL}$ of the equilibrated solution was added to $8.5 \mathrm{~mL}$ of ultrapure water with a resistivity of $18.2 \mathrm{M} \Omega \mathrm{cm}$ as a final dilution step. Earlier studies demonstrated that uranyl peroxide cage clusters remain intact subsequent to this dilution [22]. Four sets of solutions were prepared. Three were spiked with $0.5 \mathrm{~mL} 0.5 \mathrm{M}$ dipotassium ethylenediaminetetraacetic acid $\left(\mathrm{K}_{2}\right.$ EDTA) while the fourth was not modified. The EDTA was added after preliminary experiments demonstrated that Cs, Sr, and Ba were not separated efficiently from the clusters based exclusively on size. The samples are denoted WW (no spike, $\mathrm{H}_{2} \mathrm{O}$ wash), EW (EDTA spike, $\mathrm{H}_{2} \mathrm{O}$ wash), EE (EDTA spike, EDTA wash), and ED (EDTA spike, diethylenetriaminepentaacetic acid (DTPA) wash) in the following discussions. The solutions were then sampled directly for original concentration values and spectroscopic data before being placed into the stirred cell for processing. 


\subsection{Ultrafiltration}

A diafiltration system was employed using a Millipore Amicon 8010 stirred cell and RC800 Mini-Reservoir in combination with a Millipore CDS10 Concentration/Dialysis Selector. In a typical experiment, the stirred cell was filled with $10 \mathrm{~mL}$ of dissolved SIMFUEL solution and the reservoir was filled with 700-900 mL of ultrapure water (WW, EW), $500 \mu \mathrm{M}$ EDTA (EE), or $500 \mu \mathrm{M}$ DTPA (ED). Reservoir solutions were adjusted to match dissolved SIMFUEL pH values ( 8.5) using LiOH. Then an applied pressure, generated using pressurized nitrogen gas, was used to drive the flow of reservoir solution into the stirred cell and through flat sheet membranes. The permeate mass across the membrane was monitored over time and recorded using a Sartorius balance linked directly to a computer. A typical experiment proceeded until the initial dissolved SIMFUEL volume (DV) had been replaced 4-8 times by reservoir solution. Permeate solution was collected in a scintillation vial to monitor concentrations of the various elements. Starting and final masses of the liquid filled stirred cell were recorded to demonstrate mass balance.

In order to minimize the influence of concentration polarization, the stir speed $(\omega)$ used during the ultrafiltration experiments was determined using the relationship developed by Zeman and Zydney for this particular stirred-cell design:

$k b / D_{i}=0.23\left(\omega b^{2} / v\right)^{0.567}\left(v / D_{i}\right)^{0.333}$

where $b$ is the radius of the stirred cell, $\omega$ is the angular velocity of the stir bar, $v$ is the kinematic viscosity of the feed solution, $k$ is the mass transfer coefficient, and $D_{i}$ is the diffusion coefficient of species $i$ [23]. The dilute solution is assumed to have the same kinematic viscosity as pure water. Values for the diffusion coefficients, $D_{i}$, are calculated from the hydrodynamic radii, $\mathrm{R}_{\mathrm{H}}$, of the uranyl peroxide nanoclusters using: 
$\mathrm{D}=k_{B} \mathrm{~T} / 6 \pi \mu \mathrm{R}_{\mathrm{H}}$

where $k_{B}$ is Boltzman's constant, $\mathrm{T}$ is the temperature, and $\mu$ is the viscosity of the solvent (water). Hydrodynamic radii have been previously determined experimentally from dynamic light scattering (DLS) measurements and represent an average particle size in solution [24]. Typical uranyl peroxide nanocluster hydrodynamic radii from dynamic light scattering range from 2-5 nm, which leads to diffusion coefficients on the order of $10^{-6}$ to $10^{-7} \mathrm{~cm}^{2} / \mathrm{sec}$ for use in the Zeman-Zydney correlation [23]. The stir speed was adjusted to maintain the ratio of the water flux to the mass transfer coefficient equal to or less than one because this helps to reduce the deleterious effects of concentration polarization.

Model HFK-328 polyethersulfone, thin-film sheet membranes (Molecular Weight Cut-Off (MWCO): 5K-PEG, typical permiability $1.5 \mathrm{~L} \mathrm{~m}^{-2} \mathrm{~h}^{-2} \mathrm{bar}^{-1}$ ) manufactured by Koch were used in these experiments. Individual experimental parameters are listed in Table 1.

\subsection{Raman spectroscopy}

Raman spectroscopy was used to monitor selected species in the dissolved and processed SIMFUEL solutions. Spectra were collected from $2 \mathrm{~mL}$ aliquots using a Bruker Sentinel system linked via fiber optics to a video-assisted Raman probe. The spectra were typically collected at $400 \mathrm{~mW}$ for $45 \mathrm{~s}$ with a $45 \mathrm{~s}$ background over a range from 80 to $3200 \mathrm{~cm}^{-1}$. 


\subsection{Small-angle X-ray scattering (SAXS)}

To evaluate the average size and shape of the uranyl peroxide clusters in solution, their scatter pattern was collected using small angle X-ray scattering. Background measurements were accumulated using ultrapure water. The sample data were collected for $3600 \mathrm{~s}$ and corrected using background subtraction and integrated over $0.5-11.5^{\circ} 2 \theta$. The DiffracPlus Nanofit software package was used for modeling.

\subsection{Inductively coupled plasma optical emission spectroscopy (ICP-OES)}

Elemental concentrations were monitored using ICP-OES. Samples were prepared by dilution into $5 \%$ nitric acid solutions. Five standards ranging from 0.5 to $40 \mathrm{ppm}$ were prepared to provide external calibration curves for evaluation of the unknown concentrations. Each standard, blank, and sample was spiked with a $1.0 \mathrm{ppm} \mathrm{Y}$ internal standard to monitor matrix effects. This internal standard was chosen after initial experiments indicated that $\mathrm{Y}$ was not present in the dissolved solutions.

Before a solution was used in a diafiltration experiment, an aliquot was removed to measure the original concentrations of the constituents of the SIMFUEL in solution. These elements were also measured in aliquots from the processed solutions and each permeate volume. This technique allows for the direct tracking of the analytes during an ultrafiltration experiment. Several analytes, including $\mathrm{Cs}^{+}$, are present in the starting material, although we experienced difficulty resolving the spectral intensity with respect to the standard curves in the original, feed, and permeate solutions. 
2.7 Inductively coupled plasma mass spectrometry (ICP-MS)

ICP-MS was utilized to obtain quantitative data for the elements not resolved using ICP-OES. Analyses were conducted using a Thermo Finnigan Element2 High Resolution ICP-MS. Samples were prepared by dilution into 5\% nitric acid solutions. Standards ranging in concentration from 0.05 to $30 \mathrm{ppb}$ were prepared to provide an external calibration curve for evaluation of the unknown concentrations. A $1.0 \mathrm{ppb}$ Tl-Bi solution was added to all standards, blanks, and samples to provide internal standardization. The sampling design was conducted in the same manner as described for ICP-OES.

\section{Results and Discussion}

Crushed SIMFUEL consisting of pressed and sintered $\mathrm{UO}_{2}$ containing non-radioactive isotopes of 16 fission products, as well as depleted uranium, rapidly dissolved in aqueous solution containing lithium hydroxide and hydrogen peroxide, leaving a small residual of insoluble material that corresponds to the oxides of specific fission products. Solutions of dissolved SIMFUEL were processed by ultrafilitration [22], and the initial and processed solutions where characterized by X-ray scattering, Raman spectroscopy, and chemical analyses. Small-angle Xray scattering (SAXS) data demonstrate that nanoscale clusters are present in both the original and processed solutions subsequent to dissolution and throughout the duration of each experiment (Fig. 2). Modelling of the data indicates these are spherical shell clusters (cages) with average inner and outer radii of 5.2 and $9.3 \AA$, respectively. These particles are similar in size 
and shape to many previously described uranyl peroxide nanocluster species observed both in solution and the solid-state $[12,13,20]$. It is likely that the clusters formed consist of a mixture of clusters such as U24 and U32, consisting of 24 and 32 uranyl peroxide hydroxide polyhedra, respectively, as these are known to form in the presence of lithium cations in solution [12].

Raman spectra collected from the original and processed solutions indicate that the bonding characteristics of the uranyl species remain relatively unaltered as a result of diafiltration (Fig. 3). Peaks centered between 805 and $815 \mathrm{~cm}^{-1}$ are assigned to the U-O uranyl shifts. Shoulders centered between 825 and $845 \mathrm{~cm}^{-1}$ are assigned to the uranyl peroxide U-O shifts [13].

Of the 17 elements of interest present in the original SIMFUEL material, five could be reliably measured in the solutions of dissolved SIMFUEL using ICP-OES techniques, while seven were measured with ICP-MS. The other constituents are not present in the dissolved fraction at levels detectable by these techniques, as they partitioned into insoluble solids during dissolution of the SIMFUEL. The Li and $\mathrm{K}$ added to the systems as a result of solution preparation were measured as well. The full set of concentration values are summarized in the Supporting Information.

Solutions of dissolved SIMFUEL were processed by ultrafilitration in a stirred-cell apparatus, as detailed in the Materials and Methods section. Retention coefficients $\left(R_{o}\right)$, presented in Figure 4, are calculated based on the concentration measured in the original dissolved fraction $\left(c_{d}\right)$ relative to the retentate concentration $\left(c_{r}\right)$

$$
R_{o}=\frac{c_{d}}{c_{r}}
$$


These data show that where pure water was used as a wash solution, $\mathrm{Sr}, \mathrm{Ba}$, Cs, and Nd are retained very efficiently while a significant amount of Mo and Te permeate through the membrane. The $\mathrm{U}$ also displays high retention values, as expected, due to the size-selectivity of the membrane for the large uranyl peroxide clusters. Stated differently, when pure water was used as a wash solution, the uranium clusters were not separated from the bulk of Sr, Ba, Cs, and Nd. As previous work demonstrated that hydrated cations $\left(\mathrm{Cs}^{+}, \mathrm{SrOH}_{\mathrm{n}}{ }^{2-\mathrm{n}+}, \mathrm{BaOH}_{\mathrm{n}}{ }^{2-\mathrm{n}+}, \mathrm{NdOH}_{\mathrm{n}}{ }^{3-}\right.$ ${ }^{\mathrm{n}+}$ ) permeate freely through membranes in this pore size regime under these solution conditions, we conclude that the cations are closely associated with the anionic uranyl peroxide cage clusters as charge-balancing species even in the presence of a large excess of $\mathrm{Li}^{+} .<$Wylie, $2014 \# 25>$ This is consistent with the large hydrated radius of $\mathrm{Li}$, which reduces its affinity with the anionic clusters.

When dissolved SIMFUEL solutions were spiked with either dipotassium ethylenediaminetetraacetic acid (K-EDTA) or diethylenetriaminepentaacetic acid (DTPA), the retention coefficients of the surrogates (Sr, Ba, Cs, Nd, Mo, Te) decreased significantly (Fig. 4). In other words, these cations passed through the membrane and, therefore, were separated from uranium. Raman spectra suggest that both free and chelating EDTA (Pr complexes) is permeable through the pore sizes used in these experiments (Supporting Information). As such, we interpret that the decreased retention (lower $R_{o}$ ) of surrogate fission products results from their chelation and transport across the membrane as EDTA/DTPA complexes. The clusters remain stable due to charge-balance with excess $\mathrm{Li}^{+}$and $\mathrm{K}^{+}$that is not chelated as effectively by EDTA and DTPA. Further evidence of this mechanism is provided by the even lower $R_{o}$ values that are observed in the experiments that use reservoir solutions containing sequestering agents relative to experiments that use a pure water reservoir solution (EE and $\mathrm{ED}<\mathrm{EW})$. 
To quantify the amount of separation in terms of mass that is attained by one cycle of the ultrafiltration process, we consider the concentration that is expected in a solution with $100 \%$ solubility of each analyte from the bulk material. Then, we compare these concentrations to an average of the ones measured from the dissolved SIMFUEL material to obtain a difference percentage. This provides information about how much of each analyte is initially dissolved (Table 2). Note that the percentage of total uranium increases from $95.24 \%$ to $98.70 \%$ with respect to the surrogate fission products as a result of dissolution. If this process is repeated using an average of the final product concentrations (EDTA spiked experiments), one can obtain information about the amount of each analyte present in the processed solution relative to the original dissolved concentrations. Here, we observe the percentage of uranium increases again from $98.70 \%$ to $99.79 \%$ with respect to the surrogate analytes as a result of processing. This results in a total uranium increase of $4.55 \%$ with respect to the surrogates. In other words, this represents a $95.59 \%$ decrease in the mass of fission product surrogates present in the processed fraction relative to the original bulk material. Table 2 summarizes the recovered percentages based on the mass of each element.

When we consider the $\mathrm{Li}^{+}$and $\mathrm{K}^{+}$that are added to the system to control $\mathrm{pH}$ and template the nanocluster species in addition to the surrogates, the total uranium increases from $91.87 \%$ in the dissolved fraction to $97.32 \%$ in the purified product.

This study indicates that SIMFUEL material can be dissolved using oxidative dissolution reactions to promote the assembly of uranyl peroxide nanoscale cluster species. These clusters can then be separated from aqueous solution using size-selective ultrafiltration techniques. To purify the uranium product with respect to the surrogate fission products associated with the clusters as charge-balancing agents, the complexing ligands EDTA and DTPA were utilized. 
These ligands decreased the retention of the soluble cations by $60-90 \%$ with respect to processing with water reservoir solutions. Through only one cycle, we achieve an average separation efficiency $>95 \%$ relative to the original bulk pellet material. As such, this represents a hybrid technique for the separation and recovery of uranium from the fission products of a used fuel material for possible reuse.

This research addresses several key areas needed to determine the evolutionary trajectory of nanoscale processing to the industrial-scale. Here, we demonstrate that we can improve on existing separation methods from an environmental perspective based on the chemical simplicity of the system and the volume reduction of liquid waste compared with liquid-liquid extraction. This also leads to economic advantages not only with the process itself but also with handling and storage of the waste material. If continued development can improve efficiency by adding iterations and optimization with used reactor fuel, then its future evolution will shift from a scientific to an engineering challenge.

Relative to actual irradiated nuclear fuel, the surrogate used here lacks intense ionizing radiation, various important fission products including iodine and technetium, and the trans-uranium actinides, of which the most abundant in irradiated fuel will be various isotopes of plutonium, americium, and neptunium [26]. It will be necessary to expand experimental studies to address the radiation, as well as the broader range of fission products and trans-uranium elements. However, an expanded experimental effort does appear to be warranted. As ionizing radiation in water causes formation of peroxide by radiolysis [27], the chemical conditions favorable for uranyl peroxide cluster persistence should remain during intense irradiation. Both technetium and iodine are expected to be present as anionic species under the aqueous conditions used in this process, which should favor their transmission through the permeable membrane and, therefore, 
separation from uranium. In the peroxide-rich environment used in this process, plutonium is likely to be in the tetravalent oxidation state [28], which is insoluble in mildly alkaline solutions and, therefore, the plutonium is expected to partition into the insoluble residue remaining after fuel dissolution. Trans-plutonium actinides will be in the trivalent oxidation state, and are also only sparingly soluble in mildly alkaline aqueous solution. More uncertainty exists concerning the redox chemistry of neptunium in such systems, and the successful synthesis of neptunyl peroxide cage clusters in water indicates neptunium substitution for uranium may occur [12], which would result in neptunium retention in the uranium product. 


\section{FIGURES}

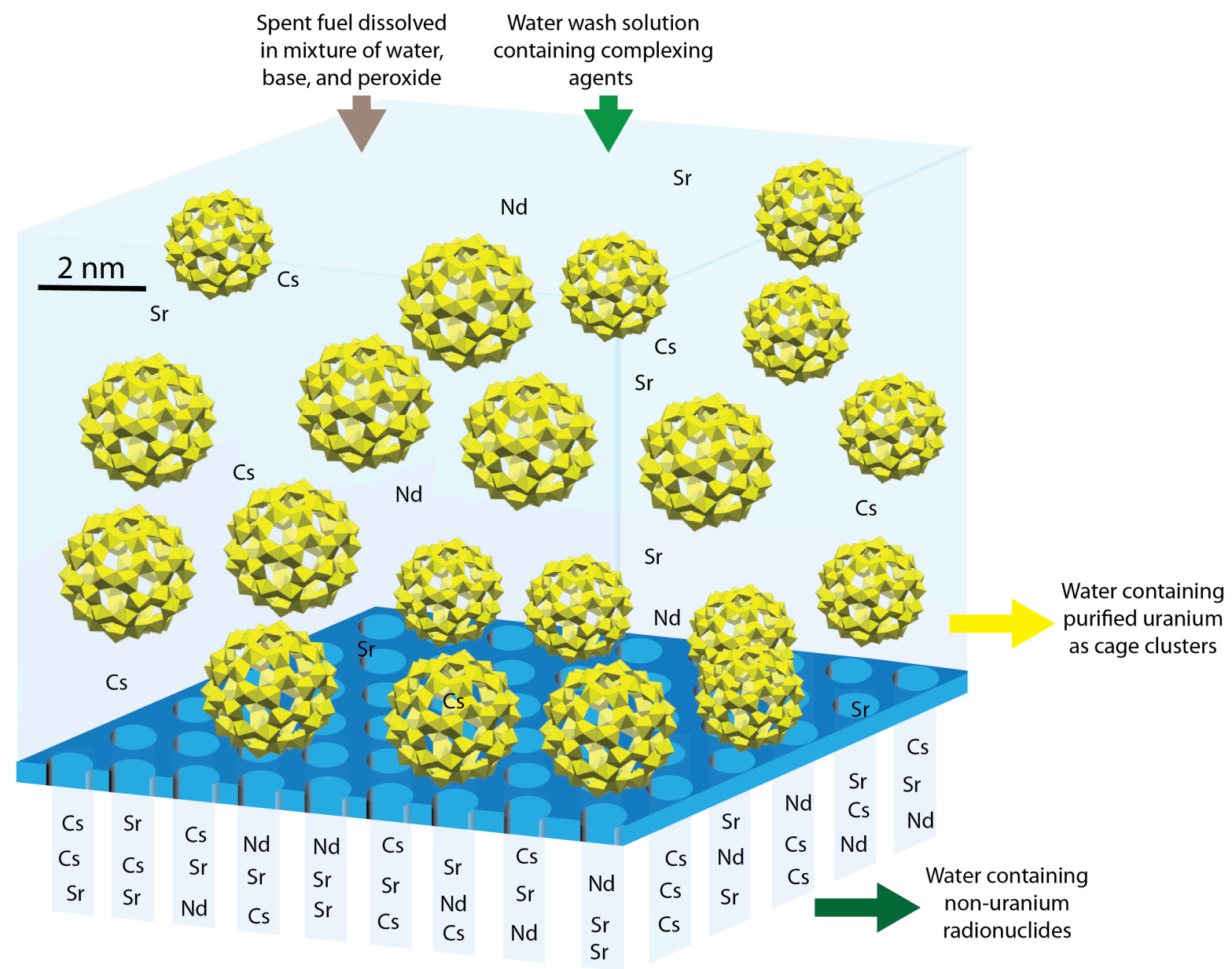

Fig. 1. Generalized illustration of the process of combining a solution containing uranyl peroxide cage clusters produced by dissolving SIMFUEL with a wash solution, followed by ultrafiltration through a porous membrane, to produce a more pure uranium product. 


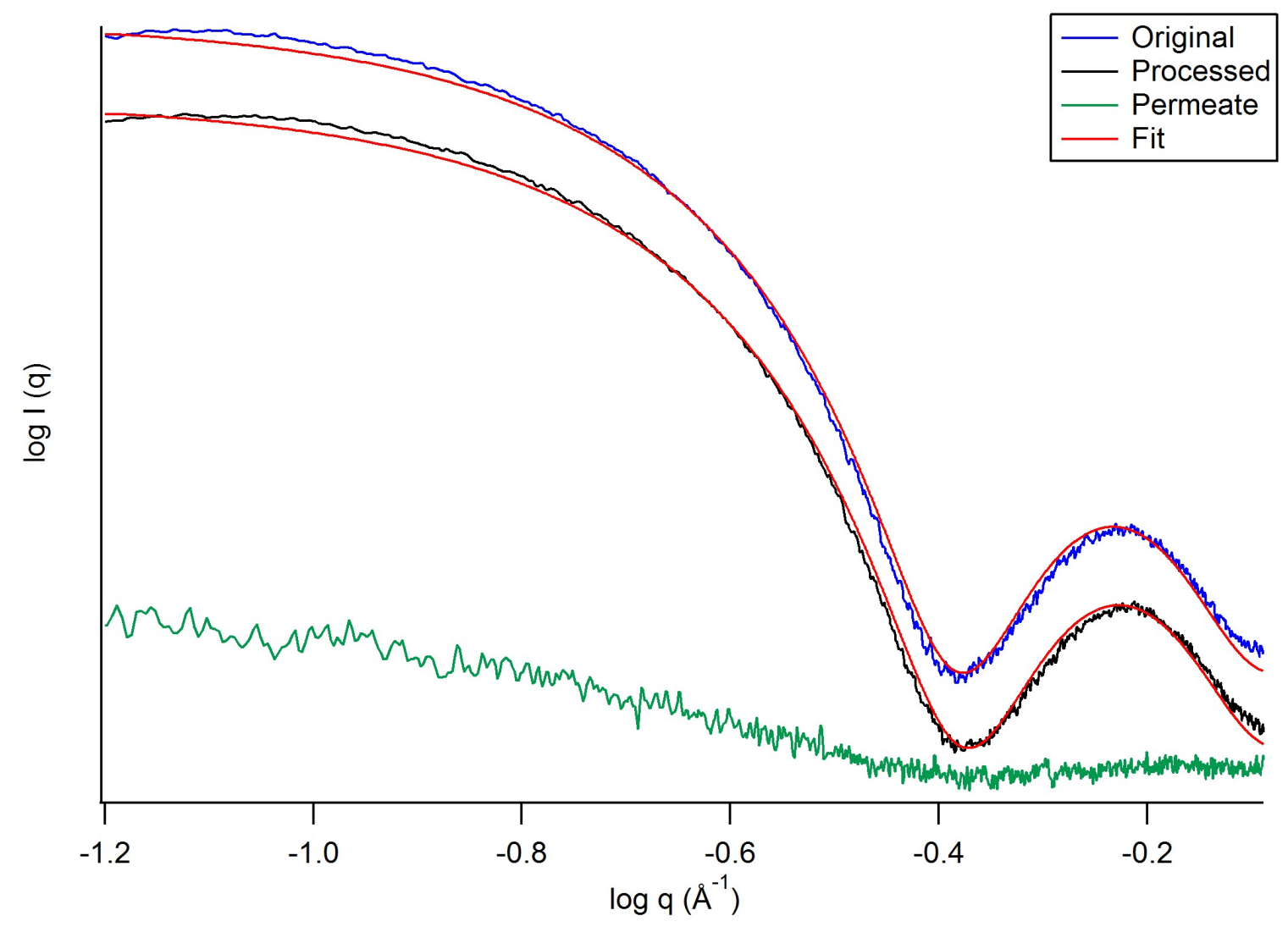

Fig. 2. SAXS patterns for a typical experiment. Original (EDTA spiked) and processed solutions (blue and black traces) are shown with their respective fit curves (red). The green trace represents a permeate solution. The intensity scale is arbitrary. 


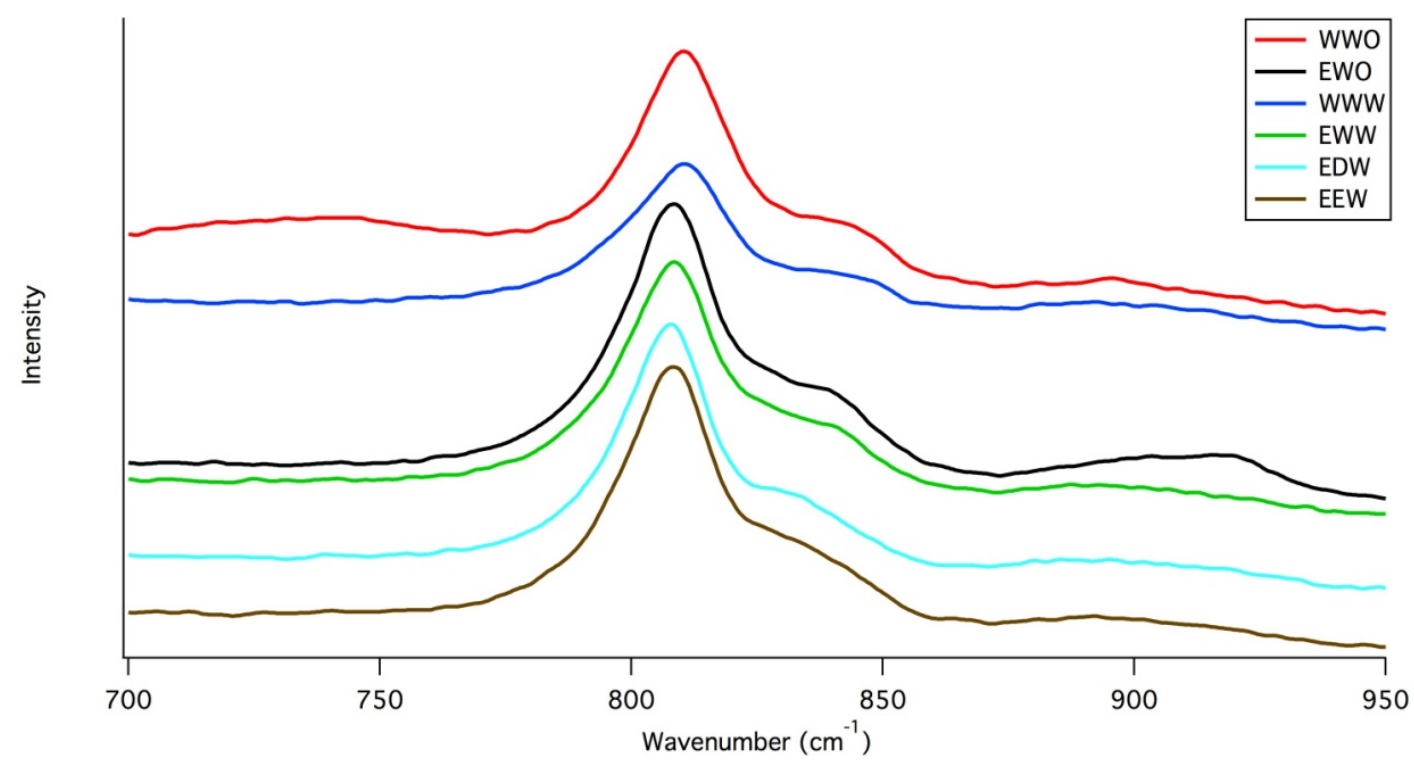

Fig. 3. Representative Raman spectra for each experiment. Labels ending in $\mathrm{O}$ and $\mathrm{W}$ represent original and processed solutions, respectively.

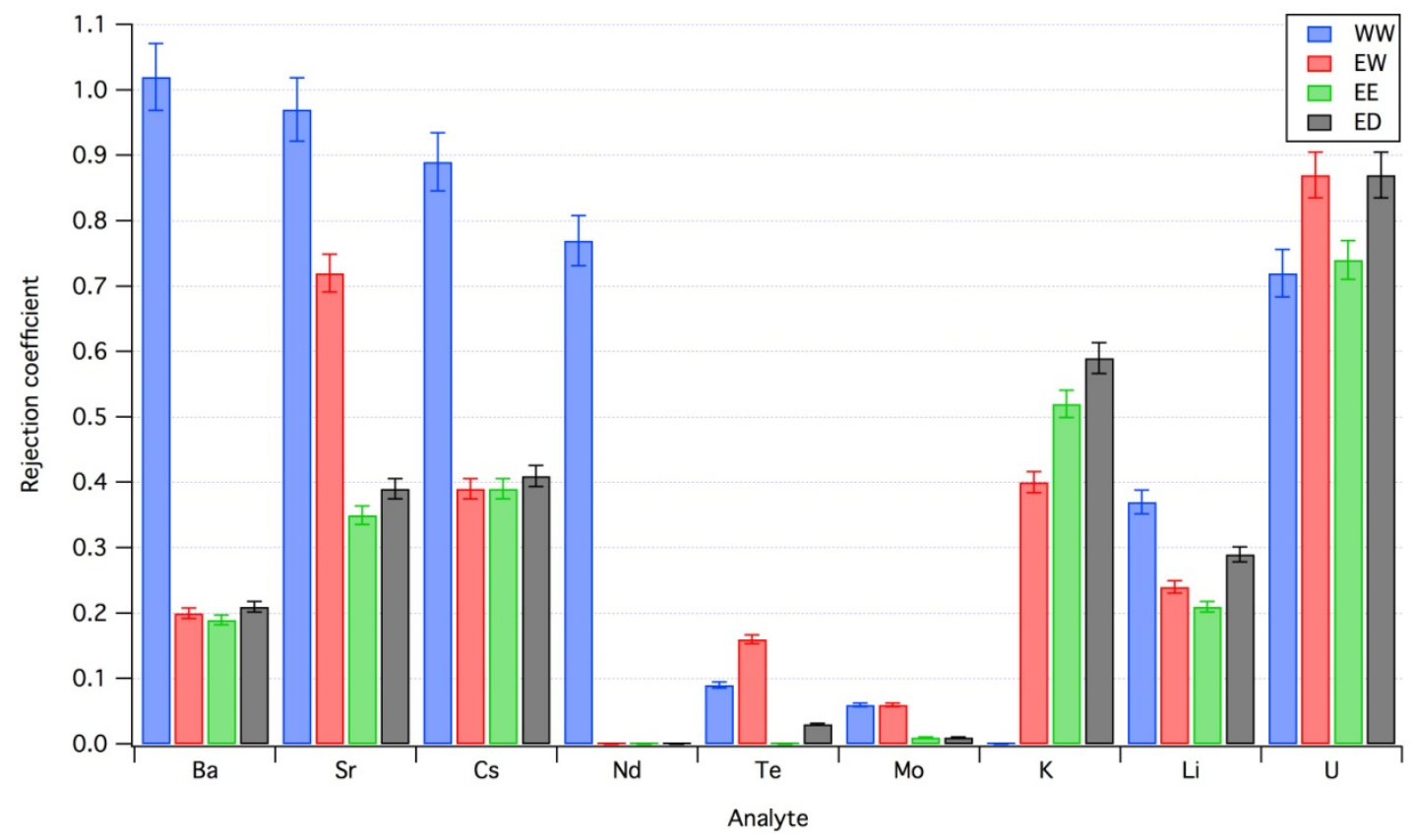

Fig. 4. Chemical analysis (ICP-OES and ICP-MS) of the soluble elements in each experiment. Rejection coefficients $\left(R_{o}\right)$ are calculated based on the concentration measured in the original dissolved fraction $\left(c_{d}\right)$ relative to the retentate concentration $\left(c_{r}\right)$. Reservoir solutions are defined as ultrapure water (WW, EW), $500 \mu \mathrm{M}$ EDTA (EE), and $500 \mu \mathrm{M}$ DTPA (ED). 
TABLES.

\section{Table 1}

Experimental parameters. Concentration factor is an average ratio of initial to final mass.

\begin{tabular}{l|llll} 
Experiment & WW & EW & EE & ED \\
\hline Reservoir Solution & QH2O & QH2O & EDTA & DTPA \\
Membrane & HFK-328 & HFK-328 & HFK-328 & HFK-328 \\
MWCO (Da) & 5000 & 5000 & 5000 & 5000 \\
Applied Pressure (psi) & 15 & 15 & 15 & 15 \\
Stir Speed (rpm) & 700 & 700 & 700 & 700 \\
Concentration Factor & 0.92 & 0.98 & 1.00 & 1.00 \\
Processed Mass (g) & 42 & 99 & 94 & 80 \\
DV Volumes Replaced & 4.8 & 11.8 & 11.5 & 9.5
\end{tabular}

\section{Table 2}

Elemental mass percentage in each experimental partition.

\begin{tabular}{ccccc} 
Analyte & \% of Pellet & \% of Dissolved & \% of Product & \% Mass Recovered \\
\hline $\mathrm{Ba}$ & 0.28 & 0.14 & 0.03 & 10.25 \\
$\mathrm{Cs}$ & 0.43 & 0.26 & 0.11 & 20.85 \\
$\mathrm{Ce}$ & 0.22 & 0 & 0 & 0 \\
$\mathrm{Eu}$ & 0.03 & 0 & 0 & 0 \\
$\mathrm{Gd}$ & 0.14 & 0 & 0 & 0 \\
$\mathrm{La}$ & 0.22 & 0 & 0 & 0 \\
$\mathrm{Mo}$ & 0.68 & 0.70 & 0.02 & 2.85 \\
$\mathrm{Nd}$ & 0.72 & 0.02 & 0 & 0 \\
$\mathrm{~Pa}$ & 0.27 & 0 & 0 & 0 \\
$\mathrm{Pr}$ & 0.20 & 0 & 0 & 0 \\
$\mathrm{Ru}$ & 0.46 & 0 & 0 & 0 \\
$\mathrm{Sm}$ & 0.03 & 0 & 0 & 0 \\
$\mathrm{Sr}$ & 0.15 & 0.11 & 0.05 & 25.28 \\
$\mathrm{Te}$ & 0.10 & 0.07 & 0.01 & 4.31 \\
$\mathrm{U}$ & 95.24 & 98.7 & 99.79 & 88.36 \\
$\mathrm{Y}$ & 0.09 & 0 & 0 & 0 \\
$\mathrm{Zr}$ & 0.73 & 0 & 0 & 0
\end{tabular}




\section{Acknowledgements}

This research is funded by the Office of Basic Energy Sciences of the U.S. Department of Energy as part of the Materials Science of Actinides Energy Frontiers Research Center (DESC0001089). Chemical analyses were conducted at the Center for Environmental Science and Technology and the Midwest Isotope and Trace Element Research Analytical Center at the University of Notre Dame. Raman spectra were collected at the Materials Characterization Facility of the Center for Sustainable Energy at the University of Notre Dame.

\section{References}

[1] K.L. Nash, Solvent Extraction and Ion Exchange 1993, 11, 729-768.

[2] K.L. Nash, G.R. Choppin, Separation Science and Technology 1997, 32, 255-274.

[3] H. Eccles, Solvent Extraction and Ion Exchange 2000, 18, 633-654.

[4] J.M. Joshi, P.N. Pathak, V.K. Manchanda, Solvent Extraction and Ion Exchange 2005, 23, 663-675.

[5] J.N. Mathur, M.S. Murali, P.R. Natarajan, L.P. Badheka, A. Banerji, A. Ramanujam, P.S. Dhami, V. Gopalakrishnan, R.K. Dhumwad, M.K. Rao, Waste Management 1993, 13, 317-325.

[6] R.G. Shuler, C.B. Bowers, J.E. Smith, V. Vanbrunt, M.W. Davis, Solvent Extraction and Ion Exchange 1985, 3, 567-604.

[7] R.C. Ewing, W. Runde, T.E. Albrecht-Schmitt, MRS Bulletin 2010, 35, 859-866.

[8] A.E.V. Gorden, M.A. DeVore, B.A. Maynard, Inorganic Chemistry 2013, 52, 3445-3458. 
[9] J.M. McKibben, Radiochimica Acta 1984, 36, 3-15.

[10] J. Bruno, R.C. Ewing, Elements 2006, 2, 343-349.

[11] P.C. Burns, Mineralogical Magazine 2011, 75, 1-25.

[12] P.C. Burns, K.A. Kubatko, G. Sigmon, B.J. Fryer, J.E. Gagnon, M.R. Antonio, L. Soderholm, Angewandte Chemie-International Edition 2005, 44, 2135-2139.

[13] J. Qiu, P.C. Burns, Chemical Reviews 2013, 113, 1097-1120.

[14] N. Asanuma, M. Harada, Y. Ikeda, H. Tomiyasu, Journal of Nuclear Science and Technology 2001, 38, 866-871.

[15] P. Govindan, A. Palamalai, T. Vasudevan, K.S. Vijayan, R.V.S. Rao, M. Venkataraman, R. Natarajan, Journal of Radioanalytical and Nuclear Chemistry 2013, 295, 77-82.

[16] C. Soderquist, B. Hanson, Journal of Nuclear Materials 2010, 396, 159-162.

[17] J. Qiu, J. Ling, L. Jouffret, R. Thomas, J.E.S. Szymanowski, P.C. Burns, Chemical Science 2014, 5, 303-310.

[18] J. Qiu, J. Ling, A. Sui, J.E.S. Szymanowski, Journal of the American Chemical Society 2012, 134, 1810-1816.

[19] J. Qiu, K. Nguyen, L. Jouffret, J.E.S. Szymanowski, P.C. Burns, Inorganic Chemistry 2013, 52, 337-345.

[20] G.E. Sigmon, D.K. Unruh, J. Ling, B. Weaver, M. Ward, L. Pressprich, A. Simonetti, P.C. Burns, Angewandte Chemie-International Edition 2009, 48, 2737-2740.

[21] A.G. Croff, ORIGEN2: a revised and updated version of the Oak Ridge isotope generation and depletion code (No. ORNL-5621); Oak Ridge National Laboratory, TN (USA), 1980.

[22] E.M. Wylie, K.M. Peruski, J.L. Weidman, W.A. Phillip, P.C. Burns, ACS Applied Materials \& Interfaces 2014, 6, 473-479. 
[23] L.J. Zeman, A.L. Zydney, Microfiltration and ultrafiltration: Principles and applications. New York, 1996.

[24] C.M. Wallace, Solution and Aggregation Behavior of the U60 Nanocluster and PostDetonation Nuclear Forensic Analysis of Trinitite. University of Notre Dame, 2013.

[25] G.E. Sigmon, J. Ling, D.K. Unruh, L. Moore-Shay, M. Ward, B. Weaver, P.C. Burns, P. C. Journal of the American Chemical Society 2009, 131, 16648-+.

[26] P.C. Burns, R.C. Ewing, A. Navrotsky, Science 2012, 335, 1184-1188.

[27] J.A. LaVerne, Radiation Research 2000, 153, 487-496.

[28] A. Morgenstern, G.R. Choppin, Radiochimica Acta 1999, 86, 109-113. 